\title{
TACITO Y LA DECADENCIA DEL IMPERIO
}

Tacitus' text, Germania XXXIII, centered around the phrase urgentibus imperii fatis, is not a totally pessimistic interpretation, despite the opinion of many contemporary writers. The Roman historian shows a critical viewpoint of the past, tied in with the relativity of time; in this way the decadence in social customs is seen as an essential part of its reflection, and therefore all his work is riddled with pessimistic annotations. His criticism is directed against the excesses of civilization, in the sense that the maintenance of the latter will be the best means the Romans have of freering themselves of the threat posed by the barbarians. Consequently, the divisions which kept the Germanic peoples in mutual discord had until that time assured the continuance of Roman power.

El capítulo XXXIII de la Germania de Tácito ha planteado buen número de cuestiones de todo tipo, a las que los investigadores del siglo actual han aportado soluciones muy dispares, en ocasiones similares y en otras contradictorias, que han contribuido a esclarecer en buena medida el sentido del mismo. El texto en cuestión afirma:

Iuxta Tencteros Bructeri olim occurrebant: nunc Chamauos et Angriuarios immigrasse narratur, pulsis Bructeris ac penitus excisis uicinarum consensu nationum, seu superbiae odio seu praedae dulcedine seu fauore quodam erga nos deorum; nam ne spectaculo quidem proelii inuidere, super sexaginta milia non armis telisque Romanis, sed quod magnificentius est, oblectationi oculisque ceciderunt, maneat, quaeso, duretque gentibus, si non amor nostri, at certe odium sui, quando urgentibus imperil fatis nihil iam praestare fortuna maius potest quam hostium discordiam 1.

Junto a los téncteros se hallaban en otro tiempo los brúcteros: se cuenta que en la actualidad los chamavos y angrivarios se han establecido en el país, una vez expulsados y completamente destruidos los brúcteros por una liga de pueblos vecinos, bien debido al odio de su orgullo, bien a la atracción del botín, bien a algún favor de los dioses hacia nosotros;

1 Tac., Germ. XXXIII. 
en efecto, ni siquiera nos han envidiado el espectáculo del combate: más de sesenta mil perecieron, no bajo las armas y los golpes de los romanos, sino, lo que es más grande, por su placer y sus ojos. Que pueda durar y persistir entre las naciones, si no la voluntad de agradarnos, al menos el odio que conllevan, puesto que a los destinos del Imperio la fortuna no puede aportar ya un regalo más bello que la discordia entre sus enemigos.

La explicación y comprensión de dicho texto incide sobre las palabras urgentibus imperii fatis, en las que se ha tratado de ver un concepto histórico totalmente pesimista por parte del historiador romano. Sin embargo, sobre dicho aspecto se han aportado interpretaciones muy variadas, que vamos a analizar a continuación.

Ya en el año $1914 \mathrm{R}$. Reitzenstein ${ }^{2}$ aseguró que el tiempo en que fue escrita la Germania constituía un momento propicio para esta afirmación de Tácito, dado que el imperialismo contó entonces con un periodo de renacimiento y el historiador se mostró como su apoyo más grande. En este contexto la uirtus romana y el fatum imperii Romani se manifestaban irresistibles, y dicho fatum aplastaría a los germanos ${ }^{3}$.

En una revisión de caracteres más reducidos que la ofrecida por Reitzenstein, G. Andresen rechazó esta interpretación y volvió nuevamente a la consideración de la interpretación de dichas palabras en el sentido de que Tácito pone de manifiesto su temor de que el edificio del Estado romano no podrá resistir la presión de un pueblo como los germanos ${ }^{4}$. Dicha interpretación sirvió, sin embargo, para convencer a Reitzenstein, quien cambió su primitiva postura en un trabajo publicado en el año $1926^{5}$. En consecuencia, para los deseos de muchos, la última palabra fue dicha por R. Heinze dos años después en un discurso que se publicó con posterioridad a su muerte ${ }^{6}$. Heinze rechaza igualmente la interpretación optimista de Reitzenstein e insiste en el hecho de que Tácito siente miedo ante el momento en que los bárbaros, uniendo sus fuerzas, atacarán el Imperio.

Estas conclusiones hallaron mucho eco en las décadas posteriores a su publicación: así, E. Wolff, por ejemplo, a través de un artículo bastante extenso ${ }^{7}$, asegura que el punto de vista del historiador al

2 «Bemerkungen zu den kleinen Schriften des Tacitus», NGG, Phil.-Hist. Kl., 1914, pp. 173-276, esp. 255-259.

3 Cf. E. Paratore, «I Germani e i loro rapporti con Roma dalla Germania agli Annales di Taciton, RomBarb 2, 1977, pp. 149-182.

4 En WKPh 32, 1915, pp. 755-758.

5 *Tacitus und sein Werk», Neue Wege zur Antike 4, 1926, pp. 1-32, esp. 15.

6 «Vrgentibus imperii fatis», Vom Geist des Römertums, 19602, pp. 255-277.

7 «Das geschichtliche Verstehen in Tacitus' Germania», $H$ 69, 1934, pp. 121-166. 
escribir la Germania era político, y no se encontraba en una situación apropiada para esbozar una pintura de la caida del mundo romano en manos de los germanos. A ello hemos de añadir una pequeña objeción por parte de R. Syme: "In the comments which this edifying spectacle has moved him to record, it is perhaps permissible to read, not so much solicitude for the future destiny of the Empire and hope that the enemies of Rome may ever divided thus, as irony and indignation that so ignoble a policy should in the present be recommended ${ }^{\text {: }}$.

Algunos años después, E. M. Sanford no cree en la tristeza o melancolía de Tácito ${ }^{9}:$ subraya el hecho de que la creencia en la inmortalidad de Roma contaba con una larga tradición en tiempos del historiador; este punto de vista fue sintetizado posteriormente por parte de E. Howald en un trabajo sobre dicho autor ${ }^{10}$. Con posterioridad J. M. C. Toynbee publicó una breve nota, que se encargó de contrarrestar lo que parecía ser ya el punto de vista general "1, tomando como base la publicación de dicha obra de Tácito unos años antes ${ }^{12}$. Las afirmaciones de Toynbee, aunque no han sido seguidas por otros investigadores posteriores, revisten una importancia crucial: intenta, como hizo Reitzenstein, encuadrar la nota de Tácito en el contexto de su época, un periodo en el que se puede encontrar con mayor facilidad confianza que desesperación. Una confrontación entre el autor y el clima político que le rodeaba se muestra esencial para poder llevar a cabo una evaluación de las palabras del historiador.

E. Paratore, en su obra general sobre Tácito ${ }^{13}$, encabeza nuevamente la vertiente pesimista y melancólica: el final del capitulo XXXIII de la Germania es la clave de toda la obra y muestra, ante todo, la angustiosa preocupación del historiador por el destino de Roma; la existencia real del Imperio se ve amenazada por los germanos.

$\mathrm{Al}$ año siguiente $\mathrm{H}$. Drexler afirmaba que la única meta de los pollticos romanos era la magnitudo, la maiestas populi romani ${ }^{14}$. En una época en que Tácito sentía que Roma estaba amenazada en el exterior por un pueblo invencible hasta entonces y en el interior por la corrupción de las costumbres, a lo que se añadía la privación de libertad, quiso mostrar cuáles eran las bases de dicha libertad: honos, dignitas y fortitudo, que se hallaban entre los germanos, dignos de oponerse a

8 CAH 11, Cambridge 1936, p. 185.

9 "Contrasting Views of the Roman Empire», AJPh 58, 1937, pp. 437-456.

10 Vom Geist antiker Geschichtsschreibung, Munich 1944, p. 199.

11 "Two Notes on Tacitus", CR 58, 1944, pp. 39-43, esp. 41-43.

12 J. G. C. Anderson, Germania, Oxford 1938.

13 Tacito, Milán 1951 (Roma 1962 ).

14 «Die Germania des Tacitus», Gymnasium 59, 1952, pp. 52-70. 
la magnitudo populi Romani. Al hacer esto utilizaba Tácito una escala de valores identificada con la moral romana en general.

El principal exponente de los investigadores que defienden la postura de que el pesimismo de Tácito está expresado en dicho capítulo de la Germania lo constituye sin discusión V. Poeschl ${ }^{15}$, para quien la concepción del historiador romano se halla basada, junto a la fidelidad al ideal de la antigua Roma, en tres hechos característicos: la convicción de que la grandeza y decadencia de un pueblo son debidas a causas morales, el pesimismo y el sentimiento de responsabilidad y culpabilidad frente a los acontecimientos. La advertencia dada por Tácito en dicho pasaje se encuentra igualmente en la tradición pesimista de la historiografía romana a lo largo de los grandes períodos de crisis, asemejándose además a numerosas alusiones a la amenaza de la destrucción del Imperio que se pueden desgranar en las Historias y los Anales ${ }^{16}$.

Una respuesta vigorosa, y de carácter negativo, al punto de vista de Poeschl nos la ofrece K. Büchner ${ }^{17}$, quien introduce un aspecto nuevo en la interpretación de dicho pasaje. Da por sentado que la emoción extrema expresada en esta noticia puede ser relacionada con los premios más altos, la fortuna del Imperio, y en este contexto la fortuna no puede ofrecer nada más significativo que la falta de unidad de sus enemigos ${ }^{18}$. Pero dicha interpretación es comprendida como referencia no a la cuestión de la existencia, sino a la de su grandeza histórica.

W. Schmid asegura, sin embargo, que existen ciertas contradicciones en Büchner en oposición a Poeschl ${ }^{19}$. Vuelve al punto de vista desarrollado por Reitzenstein, quien ve en el texto de Tácito un pasaje paralelo con el de Lucano (Farsalia X 28 ss.). En este mismo sentido ya otros autores ${ }^{20}$ habian observado con anterioridad el paralelo existente entre el juicio emitido por el historiador romano y el que aparece en Polibio (XI 34) acerca de la anarquía bárbara.

15 «Die römische Auffassung der Geschichte», Gymnasium 63, 1956, pp. 190-206.

16 V. Pöschl, *Tacitus und der Untergang des römischen Reiches», WS 69, 1956 (Festschrift Lesky), pp. 310-320.

17 -Hat Tacitus geglaubt, der Untergang des römischen Reiches stehe ummittelbar bevor?», Festschrift W. H. Schuchardt, Baden-Baden 1960, pp. $43-48$.

18 J. Lacroix, «Fatum et fortuna dans l'œuvre de Tacite», REL 29, 1951, pp. 247. 264.

19 \&Vrgentibus imperii fatis (Tac., Germ. 33)», Studies Albareda, Nueva York 1961, pp. 381-392.

20 Cf. L. Alfonsi, «Su un passo della Germania e sulla sua traduzione», Aevum 27, 1953, p. 260, y «Problematicità della Germania tacitiana», Mélanges P. Boyancé, Roma 1974, pp. 5-19. 
$\mathrm{Al}$ año siguiente $\mathrm{B}$. Zanco ${ }^{21}$ llega a la conclusión de que dicho pasaje de Tácito debe de ser comprendido en el sentido siguiente: «mientras que los destinos del Imperio preparan una época difícil, de desastres inminentes». En un contexto un poco diferente U. Knoche asegura la creencia del historiador romano en la cualidad eterna del Estado romano ${ }^{22}$; su desesperación, desprendida de sus experiencias en tiempos de Domiciano, se vio contrabalanceada por su esperanza puesta en Trajano. En consecuencia existía una tensión entre el pasado, por un lado, y el presente y el futuro, por otro.

Esta reacción contra el punto de vista del pesimismo de Tácito continuó manifestándose en otros autores, como W. Steidle por ejemplo ${ }^{23}$ : acerca la interpretación del capítulo XXXIII de la Germania al resumen histórico que aparece en el XXXVII y señala que en este último no existe signo alguno de la creencia de que la existencia de Roma se halla amenazada. El Imperio romano no sólo no se enfrenta de un modo pasivo a sus enemigos, sino que, como Agrícola pensó en lo relativo a la conquista de Irlanda, se muestra capaz de llevar a cabo la expansión imperial. Así pues, la aniquilación de los brúcteros no se considera tanto una ayuda para los romanos como la base de una gran satisfacción.

Una reacción contraria, en la que se vuelve nuevamente a la posición expuesta por Poeschl, nos es presentada por R. Häussler ${ }^{2}$; sin embargo, no presupone una decadencia del Imperio romano en tiempos de Trajano. No hay duda, en la apreciación de dicho autor, de que el Imperio ha visto ya sus mejores días y ha pasado su cénit, lo que puede estar implícito en las palabras nihil iam maius. Cuando Tácito contemplaba las circunstancias históricas, el gobierno y los ejércitos de su tiempo no fue capaz de prever una gran hazaña.

Poco tiempo después A. W. Benario ${ }^{25}$ plantea el problema de nuevo: ¿el texto de la Germania se manifiesta como expresión del pensamiento de su autor o, más bien, de una visión del destino de Roma? Resulta abusivo para él atribuir al historiador romano una actitud sistemáticamente pesimista; en la Germania y la Vida de Agrícola la atmósfera que se respira no es la misma que en las Historias y los Anales; en realidad Tácito celebra la vuelta de la libertad y observa el porvenir con

21 «ota sull'interpretazione di urgentibus imperii fatis, Tacito, Germania XXXIII 3*, Aevum 36, 1962, pp. 529-531.

22 *Zur Beurteilung des Kaisers Tiberius durch Tacitus», Gymnasium 70, 1963, pp. 211-226.

23 *Tacitusprobleme», $M H$ 22, 1965, pp. 81-116, esp. 88-95.

24 Tacitus und das historische Bewusstein, Heidelberg 1965, pp. 264266 y 279-280.

25 .Tacitus and the Fall of the Roman Empire*, Historia 17, 1968, pp. 37-50. 
confianza, teniendo en cuenta los grandes acontecimientos pasados y sin obnubilarse ante la perspectiva de los desastres cercanos. Al comienzo de su carrera como historiador Tácito comparte con el pueblo romano la confianza que dio origen al beatissimum saeculum del emperador Trajano ${ }^{20}$.

Igualmente por entonces retomó el problema K. Kraft ${ }^{n \text { : las pala- }}$ bras urgentibus fatis poseen para él, en este contexto, al igual que en otros autores antiguos y en especial en Lucano (X 30), un valor negativo, sin evocar en ningún caso la idea de expansión del Imperio; sin embargo, Tácito no piensa en una crisis real, inminente, sino en la eventualidad de una guerra civil, que se consideraba posible siempre en Roma.

De esta misma época arranca el análisis, por parte de algunos historiadores actuales, del problema de la crisis o decadencia romana no sólo en Tácito, sino con anterioridad en Salustio: la conjuración de Catilina, la guerra de Yugurta y los sucesos narrados en las Historias constituyen para Salustio ejemplos que ilustran la situación política de su época ${ }^{23}$. Junto a la formulación de la crisis de la República romana se sitúa igualmente la de la decadencia del Imperio en Tácito ${ }^{20}$.

Para otros investigadores posteriores el pensamiento del autor en el pasaje que estamos considerando revela la convicción de una contracción inherente a los hechos, y con el fin de dar explicación a la realidad el historiador romano recurre a un método casuístico ${ }^{30}$ : al reflexionar acerca de la historia de Roma separa las condiciones necesarias para su objetivo. El análisis del mecanismo de las guerras civiles, así como del desarrollo entre los hombres de la pasión de poder, le proporcionaban razones de temor. Sin embargo, el análisis y estudio del peligro representado por los bárbaros le llevaba, por el contrario, al optimismo debido al carácter crónico y a su lógica desunión interna.

En cuanto a M. Erren ${ }^{31}$, tras estudiar la historia de la controversia y el análisis correspondiente de las palabras fatum y urgere, así como de la expresión urgentibus fatis en los diferentes pasajes de los autores latinos, le lleva a concluir que, en este pasaje del capítulo XXXIII de

\footnotetext{
26 A. W. Benario, An Introduction to Tacitus, Georgia 1975.

27 «Vrgentibus imperii fatis, Tacitus, Germania 33», H 96, 1968-1969, pp. 591-608.

28 G. Perl, «Sallust und die Krise der römischen Republik», Philologus 113, 1969, pp. 201-216.

29 E. Koestermann, «Das Problem der römischen Dekadenz bei Sallust und Tacitus», ANRW (Festschrift Vogt), Berlín-Nueva York 1973, I 3, pp. 781-810.

30 M. P. Delpuech, «Vrgentibus imperii fatis. Tacite et la fin de l'Empire», Association G. Budé. Actes IXe Congrès, Paris 1975, pp. 995-1018.

31 *Vrgentibus imperii fatis», LF 99, 1976, pp. 1-30.
} 
su Germania, Tácito presenta efectivamente la discordia de los enemigos como el mejor regalo que la fortuna podía hacer a los romanos. Este don precioso lo lleva a cabo bajo la presión del fatum, que había destinado al Estado romano hacia una expansión brillante y victoriosa 32. En el momento en que el historiador romano llevó a cabo la redacción de este pasaje de su obra le es perdonado el haber dado tal previsión de los hechos, que se revelaría errónea en los años siguientes.

Finalmente ha tratado de nuevo este mismo problema el historiador francés A. Houlou ${ }^{33}$, recogiendo las diversas hipótesis planteadas en torno a dicho texto, así como los argumentos ofrecidos en ambas direcciones: el pesimismo de Tácito y la visión del destino de Roma.

La única obra distinta del corpus taciteo que puede compararse con la Germania es el Agricola, contemporáneo de ella, puesto que ambas pueden ser consideradas como la expresión de la política exterior romana. Parece existir, en efecto, en el texto de Tácito que estamos analizando una contradicción fundamental entre el pesimismo de la frase, en la que parece aventurarse el final del Imperio, y la confianza que se expresa el mismo año en el Agrícola; de acuerdo con ello algunos investigadores actuales, sin tener en cuenta la época, no han dudado en afirmar que el historiador romano preveía ya el final, cercano e ineludible, del Imperio romano ${ }^{34}$.

Para poder comprender el sentido de la expresión de Tácito se hace necesaria una meditación profunda acerca de la formulación de la decadencia y de la forma del tiempo. Por una parte se hace del historiador romano el censor desesperado de una decadencia irreversible y por otra se está de acuerdo en considerarlo partidario de una concepción cíclica del tiempo, a pesar de que una decadencia lineal no puede ir emparejada con un tiempo cíclico. Tácito era el heredero directo de una larga e intensa tradición tanto griega como latina: la teoría de la decadencia tiene su origen ya en Heródoto en Grecia y en Fabio Píctor entre los romanos ${ }^{35}$. Dicha teoría se puede seguir con facilidad en los autores romanos posteriores: en primer lugar en Tito Livio ${ }^{36}$, y posteriormente en Salustio ${ }^{37}$ y Veleyo Patérculo ${ }^{38}$.

32 También presentan los fata en función de la fortuna otros autores latinos: cf. W. H. Friedrich, «Cato, Caesar und Fortuna bei Lucan», H 73, 1938, p. 408.

33 «Vrgentibus imperii fatis: à propos d'un passage controversé de Tacite, Germanie 33x, Centre J. Palerne. Mémoires, I, Saint-Etienne 1978, pp. 59-66.

34. E. Paratore, op. cit., pp. 318-335.

35 FGH $809 \mathrm{~F}$ 27. Cf. S. Mazzarino, Il pensiero storico classico, Bari 1966, II 1 , p. 464 ss. y II 2, p. 247 ss.

36 Praef. 4 y $9-12$.

37 Cat. $\mathbf{X}$.

38 II 1, 1. Cf. M. P. Delpuech, op. cit., p. 996. 
En el terreno estrictamente político la idea de la decadencia ineludible y de la corrupción de los regímenes y constituciones, definida por Platón en los libros VIII y IX de la República con sus corolarios de orden económico y moral ${ }^{39}$, fue tomada de nuevo en el pensamiento antiguo a través de textos que resultan muy conocidos en este sentido ${ }^{00}$. No resulta, pues, extraño que encontremos en Tácito numerosos restos de este mismo planteamiento: así, en sus pasajes acerca de los progresos de la discordia entre los ciudadanos y el desarrollo de la lucha por el poder, ligados a la expansión del Imperio ${ }^{41}$, o en el que se refiere a los distintos tipos de constitución ${ }^{2}$, así como, en cierta medida, en el texto en el que se describe la historia del derecho ${ }^{43} \mathrm{o}$, finalmente, en el texto en que Asinio Galo trata de hacer un elogio del lujo mostrando que se manifiesta inseparable del engrandecimiento de Roma ${ }^{4}$. Todos ellos constituyen reflexiones históricas sobre la evolución de Roma desde sus orígenes, mostrándose Tácito tributario de sus antecesores, que habían dado origen al planteamiento de esta problemática.

En general podemos afirmar que nuestro autor toma en consideración un punto de vista crítico sobre el pasado, que resulta bastante destacable y no tiene equivalente alguno más que en Séneca ${ }^{45}$ : se trata de una costumbre de pensamiento, que se halla igualmente puesta en cuestión, en principio en el campo filosófico y posteriormente en el histórico. Dicha innovación, considerable en el conocimiento histórico, se encuentra ligada en su punto de partida a una intuición muy moderna de la relatividad del tiempo ${ }^{*}$. Sin embargo, el historiador romano parece creer que es posible un progreso, lo que está corroborado a través de situaciones en las que se inclina hacia una debilitación de

\footnotetext{
39 Rep. II 269 b - 374 c.

40 Polyb. VI 5 ss. y Cic., De Rep. I 67. Cf. A. Michel, «Ordres et classes chez les historiens romains», Recherches sur les structures sociales de l'Antiquité classique, Paris 1970, pp. 252-253.

41 Hist. II 38.

42 Ann. IV 33.

43 Ann. III 26-28. Cf. A. Michel, «Tacite a-t-il une philosophie de l'histoire?», StudClas 12, 1970, pp. 108-110.

44 Ann. II 33.

45 Ben. I 10, 1 y $N$. Q. V 15, 2: Intellexi enim saeculum nostrum non nouis uitiis sed iam inde antiquitis traditis laborare. Cf. N. Santos, «La concepción de la historia de Roma como sucesión de edades en los historiadores latinos», CFC (en prensa).

46 A. Momigliano, *Time in Ancient History*, Quarto contributo alla storia degli studi classici e del mondo antico, Roma 1969, pp. 13-41, esp. 33 ss., y E. Cizek, «La structure du temps et de l'espace dans l'Agricola de Tacite», Helikon 8, 1968, pp. 238-249.
} 
la severidad, como cuando relata la posición de Peto Trásea prefiriendo para Antistio el destierro o relegación a la pena de muerte prevista por el mos maiorum".

En consecuencia, no podemos considerar a Tácito como el laudator temporis acti que se ha querido ver en él, a pesar de que no critica el pasado ni aprueba sin reservas el presente ${ }^{4}$. Es más, libre del peso de la tradición, está dispuesto a una reflexión nueva y personal acerca de la decadencia y el tiempo: de esta forma, lejos de tratarla con relación a un pasado idealizado, se esforzará por analizarla a partir de un pasado real y considerado en su justo valor. Al estudiar la concepción de la decadencia en el historiador romano, el Diálogo de los oradores se impone como punto de partida: en principio hemos de destacar el hecho de que la depauperación de la elocuencia se produce como consecuencia de un progreso político, la llegada al Principado, del que Materno hace el elogio 4.

La decadencia de las costumbres se manifiesta como el segundo aspecto esencial de dicha reflexión; en este sentido toda la obra del escritor se encuentra sembrada de anotaciones pesimistas sobre este aspecto: así, en el pasaje en que muestra que la decadencia no es lineal ni ineluctable ${ }^{50}$. Además, no todo era perfecto en el pasado, al tiempo que el presente ofrece igualmente buenos ejemplos de virtud; en este aspecto se encuadra el pasaje en el que Tácito representa a las leyes como destinadas a reprimir el avance del vicio: tras la edad de oro primitiva, la historia de la legislación muestra, al hilo de una cierta decadencia, períodos nefastos compensados por buenas iniciativas, como la Ley de las XII Tablas o las legislaciones de Sila, Augusto y Tiberio ${ }^{51}$.

No obstante, la decadencia se halla contrabalanceada por un progreso, puesto que es ocasionada por la extensión del Imperio, como lo subraya, de acuerdo con Tito Livio, Salustio y otros autores, Tiberio 52: esta expansión romana entrafia igualmente la decadencia reli-

47 Ann. XIV 48. Cf. R. S. Rogers, "The Tacitean Account of a Neronian Trial», Studies presented to D. M. Robinson, Saint-Louis 1953, II, pp. 711-718, y Ch. S. Rayment, «Political Criminal-Informer in Fact and Fiction», $C B$ 34, 1958, pp. 37-39 y 43.

48 Cf., como ejemplos, Hist. II 69, y Ann. III 51, XII 6, XIV 2 y XVI 5.

4 K. Barwick, Der Dialogus de oratoribus des Tacitus. Motive und Zeit seiner Entstehung, Berlín 1954, y A. Köhken, «Das Problem der Ironie bei Tacitus», MH 30, 1973, pp. 32-50.

${ }^{30}$ Ann. III 55. Cf. F. R. D. Goodyear, «Cyclic Development in History: a Note on Tac. Ann. 3.55.5x, BICS 17, 1970, pp. 101-106.

51 Ann. III 26-28.

52 Ann. III 54 y II 33. 
giosa y la destrucción del espíritu ciudadano ${ }^{33}$. Por último, incluso en el caso de que el ejército se deje llevar a la indisciplina y la incorporación masiva de tropas auxiliares extranjeras entrañe peligros de sedición, no existen rastros de un sentimiento de decadencia militar en Tácito: aun en los períodos más oscuros quedaban generales valerosos, como Agrícola en tiempos de Domiciano o Corbulón en época de Nerón.

A pesar de todo, el historiador romano se hace a la idea de una permanencia, a través de la misma decadencia, de los valores esenciales de Roma: lo que servía de base al Estado romano para su superioridad en el pasado no era lo que la fundamentaba en el presente. De esta forma, el mos maiorum y la libertas ${ }^{54}$, ligados a la elocuencia, han cedido su lugar, mientras que otros valores han tomado el relevo: entre ellos sobresale el Principado, con su coherencia política superior a la de la democracia y la esperanza de un optimus princeps, así como la civilización $\mathrm{y}$, en todos los casos, la uirtus individual, indispensable e inalterable ${ }^{55}$. Dicha idea de permanencia constituye la tesis aportada por Tácito a su constatación de la contradicción inherente a las cosas: no existe ni el bien absoluto ni el mal absoluto.

Esta concepción de la realidad histórica fundamentará la visión tacitea de la historia. El pensamiento predominante en la época del historiador era el de un tiempo cíclico; transferida de la cosmología a la historia, esta concepción del tiempo no implicaba, por lo general, una identidad perfecta de los diversos ciclos entre sí, sino simplemente la idea de una vuelta periódica de hechos en el curso de la historia (la concepción cíclica del tiempo histórico) ${ }^{\text {\$6 }}$. El texto más significativo de Tácito lo constituye el pasaje en el que trata de estudiar las causas de la evolución de las costumbres, y de manera especial del desarrollo y decadencia del lujo ${ }^{5}$; se trata, en efecto, de un espacio de tiempo bastante restringido, que no coincide con el otro ciclo que podemos denotar en su obra, el ciclo político que había desembocado en el Principado, forma política cercana a la antigua monarquía ${ }^{58}$.

53 Sobre la decadencia de las costumbres cf. A. Salvatore, «L'immoralité des femmes et la décadence de l'empire selon Tacite», LEC 22, 1954, pp. 245-269.

54 W. Liebeschuetz, "The Theme of Liberty in the Agricola of Tacitus», CQ 16, 1966, pp. 126-139; J. Straub, «Imperium-Pax-Libertas», Gymnasium 84, 1977, pp. 136148, y M. Ducos, «a liberté chez Tacite, droits de l'individu ou conduite individuelle? , BAGB, 1977, pp. 194217.

5s Agr. 42: Sciant quibus moris est inlicita mirari posse etiam sub malis principibus magnos uiros esse.

56 S. Mazzarino, Il pensiero storico classico, Bari 1966, II 2, p. 349 ss.

5 Ann. III 55.

58 Hist. I 84 y Ann. IV 33. Cf. N. I. Barbu, «Concetti di Tacito sulla morale e sulla politica», QU, 1974, pp. 121-130. 
En otro pasaje desarrolla el historiador una visión del tiempo implícita en su reflexión acerca de la decadencia ${ }^{59}$, al tiempo que hemos de tener presentes otros textos que muestran que el Imperio ha evolucionado desde el regnum de los antiguos reyes hasta el Principado actual, dos regímenes semejantes pero en modo alguno idénticos ${ }^{60}$.

La reflexión de Tácito sobre el final del Imperio se inscribe en el cuadro siguiente: la historia no obedece a un ciclo definido y la decadencia no es lineal; en consecuencia el Imperio no está destinado a obscurecerse ineludiblemente al final de dicho ciclo, sino que, por el contrario, la concepción cicloidal permite integrar a las diversas variaciones cíclicas en el seno de la historia imperial romana y pensar en la eternidad para el Imperio ${ }^{61}$ : esto es lo que proclaman Tiberio ${ }^{62}$ y Otón ${ }^{63}$, encontrando al mismo tiempo ciertos ecos de dicha tesis en el Agricola ${ }^{64}$.

Este tipo de planteamiento se encuentra igualmente en numerosos autores romanos (Virgilio, Tibulo, Tito Livio...), pero otros pensadores, o estos mismos, añadieron ciertos rasgos de inquietud: Polibio, por ejemplo, inicia su famosa reflexión tomando como base la caída de Roma bajo su propio peso ${ }^{6 s}$, perspectiva que se halla con relativa frecuencia en Tácito ${ }^{6}$; por consiguiente, aunque el futuro no era para desesperarse, tampoco estaba asegurado, de manera que el historiador contaba a la vez con razones para desesperar y temer, a menudo entremezclados ambos sentimientos, lo que no debe producirnos sorpresa.

La misión civilizadora e ideológica universal de Roma le garantiza una supervivencia que otros Imperios no supieron conservar. La fuerza de las armas se hace ciertamente necesaria ${ }^{67}$, pero no es suficiente. El poder de Roma estribaba precisamente en haber sabido reconciliarse

9 Hist. IV 73-74: discurso de Petilio Cerial ante tréviros y lingones para justificar el dominio romano.

60 Hist. I 84 y Ann. I 1: Vrbem Romanam a principio reges habuere; libertatem et consulatum L. Brutus instituit... Lepidi atque Antonini arma in Augustum cessere, qui cuncta discordiis ciuilibus fessa nomine principis sub imperium accepit. Cf. E. Welskopf, «Die Kunst der Suggestion in der Darstellungsweise des Tacitus», StudClas 3, 1961, pp. 361-368, y H. W. Benario, *Tacitus and the Principate», CJ 60, 1964, pp. 97-100.

61 M. P. Delpuech, op. cit., p. 1006.

62 Ann. III 6.

63 Hist. I 84.

A XXXII 9. Cf. W. Edelmeier, Tacitus und die Gegner Roms, Diss. Heidelberg 1964, pp. 11-53; W. Fauth, «Die Missgunst Roms», Anregung, Zeitschr. f. d. Höhere Schule 5, 1967, pp. 303-315.

65 VI $57,2$.

¿ Hist. I 11, 30, 50 y 80; II 31 y 76; III 55 y 77 , y IV 13 y 54 .

67 Ann. XV 1. 
durante todas las épocas con sus enemigos ancestrales, integrándolos en la vida del Imperio, y convertirlos en aliados más que en sometidos, en iguales más que en inferiores, ligando por consecuencia su destino al propio del Imperio romano ${ }^{68}$.

Esta posición por parte de Tácito implica abundantes e importantes consecuencias: en primer término la supervivencia del Imperio depende más de la política exterior de asimilación de las élites y de integración de los provinciales en la vida política de Roma, es decir, de la universalización por parte de Roma de su cultura y derecho, que del mantenimiento de las costumbres antiguas. Se afirma, además, la idea de que el peligro no proviene tanto del exterior del Imperio como de su interior; y, sobre todo, se hace evidente toda una concepción de la civilización romana comprendida como instrumento perdurable de la política exterior del Estado. Nos encontramos, de este modo, ante la principal justificación moral del imperalismo romano según Tácito, que en buena parte se halla formulada ya en Cicerón ${ }^{\phi}$. Su crítica no se dirige más que a los excesos de la civilización, lo que nos lleva a pensar que el historiador romano prefería la civilización, incluso aunque estuviese corrompida, a la grosería salvaje de los bárbaros tal como nos es descrita en la Germania.

En consecuencia, Roma cuenta con una misión civilizadora importante, reemplazando la guerra por la paz, la necesidad por la emulación ${ }^{70}$ : esta actividad es lo que constituye la grandeza de Roma y justifica su imperialismo; al mismo tiempo su mayor logro versará en llegar a dominar el Imperio por medio de la fuerza de su cultura únicamente. Sin embargo, a esta parte de ideal se añade un cálculo más realista: la fuerza de los bárbaros reside principalmente en su libertad y barbarie; pero, al contacto con la civilización, se dejan arrastrar por la molicie de los vicios romanos, cayendo en una forma más discreta, pero también más eficaz, de servidumbre ${ }^{71}$. En realidad no se trata de una crítica contra la civilización, sino de una constatación, dado que Agrícola no actúa quizás de manera humanitaria y desinteresada y, por otra parte, el efecto corruptor de la presencia de la civilización romana entre los bárbaros no es presentado como una consecuencia de la intención del general romano, sino como algo independiente de su voluntad.

68 A. Michel, La philosophie politique d Rome d'Auguste a Marc-Aurèle, París 1969, pp. 84-85.

60 De Rep. III 34-36; Phil. VIII 11; De prou. cons. 19... Cf. A. Michel, Tacite et le destin de l'Empire, Paris 1966, p. 213 ss.

0 Agr. 21, 1.

71 Agr. 21, 2: Idque apud imperitos humanitas uocabatur cum pars seruitutis esset. Cf. A. Michel, La philosophie..., p. 220. 
Del texto anteriormente reseñado parece deducirse que el medio más seguro del que disponían los romanos para desembarazarse del peligro que constituían los bárbaros era inoculando esta civilización, de la que pasaban a ser beneficiarios y víctimas a un mismo tiempo. Esto podría suponer en cierto modo la consagración de la decadencia de Roma, si el historiador contemplara en ello la única forma con la que contaba el Imperio para protegerse de los bárbaros; sin embargo, conservaba su fe intacta en los ejércitos romanos.

No obstante, no son sólo los romanos quienes imbuyen de sus vicios a los bárbaros, sino que éstos ceden igualmente ante ellos por una especie de inclinación natural; de esta forma se establecerá un ambiente de cooperación entre colonizados y colonizadores, que es exaltado por los partidarios de Agripa y constituye el fondo del discurso de Cerial ${ }^{72}$. La civilización no es perfecta: casi de manera inevitable desemboca en el lujo y la molicie, en su forma más extrema y corrompida. Pero dicho mal no constituye a su vez más que un mal menor, ya que, ablandando a los bárbaros del mismo modo que a los romanos, la misma civilización que pierde a Roma la salva. Nos hallamos ante un valor nuevo, completamente diferente del mos maiorum, sobre el que se apoya sin embargo la perennidad del Imperio ${ }^{73}$.

El mismo dilema se extiende a los valores que fundamentan el peligro bárbaro ${ }^{74}$. Según Tácito el Estado romano contaba con dos enemigos principales, cuya amenaza parecía incidir sobre la existencia del Imperio: los partos y los germanos. El propio historiador ha comparado ambos peligros y llega a la conclusión de que no hay que tener ningún miedo con respecto a los partos ${ }^{75}$. De esta forma, el peligro parecía proceder del Norte, pero el historiador subraya que una sola nación sería incapaz de eximir del yugo al universo ${ }^{7 \%}$; si el peligro debía resultar de la llegada de los germanos, éste no podría producirse más que como consecuencia de una unión de los diferentes pueblos germánicos. Todas las virtudes de dichas poblaciones culminan en una sola que las hace posibles, la libertad.

Sin embargo, esta misma libertad lleva implícito en sí el vicio perjudicial de la indisciplina y la discordia ${ }^{\pi}$. A pesar de todo, detrás de

72 Hist. IV 65 y 73-74. Cf. Ann. XI 24.

73 M. P. Delpuech, op. cit., p. 1010.

74 K. Kraft, «Vrgentibus imperii fatis...», pp. 598-601.

75 Germ. XXXVII 3: Quippe regno Arsacis acrior est Germanorum libertas. Cf. M. P. Delpuech, «Entre l'offensive et la démission: Corbulon et l'impérialisme tacitéen*, Colloque sur l'Idéologie de l'imperialisme romain, Dijon 1972, pp. 91-107.

76 Hist. V 25.

$\pi$ Germ. XXX 2, Hist. IV 69, Ann. II 44 y XIII 57, y Agr. 30, 1 y 32, 1.

L, 1.0. -3 
la discordia se muestra amenazadora la imagen de una concordia que podría llegar a conciliar a todas las poblaciones que vivían más allá del Rhin, o incluso a todos los bárbaros. En un primer momento se da cuenta Tácito de que los bárbaros resisten ante Roma, que quiere esclavizarlos, pero no poseen ninguna razón para atacar a un Imperio que les daría la espalda, salvo de manera aislada ${ }^{78}$, lo que hace que dichas incursiones esporádicas no sean consideradas peligrosas. De ahí que el historiador haga destacar con satisfacción que los germanos, desde el momento en que se les deja en paz, vuelven al punto a sus discordias continuas ${ }^{79}$.

En este contexto podemos comprender mucho mejor la descripción de la muerte de los brúcteros realizada por Tácito en el capítulo XXXIII de la Germania. De esta manera, aprovecharse de la discordia de los enemigos y desear que perdure no constituye el indicio de una degeneración del poder romano, sino, por el contrario, el resurgimiento del eterno principio de la política exterior de Roma ${ }^{80}$. Por ello el deseo que formula el historiador al final del fragmento de la obra que estamos analizando no tiene nada de llamada desesperada, de súplica angustiosa; no expresa la idea de que, sin esta discordia germánica, el Imperio correría derecho a la ruina.

Tomando como base estas perspectivas se aclara la fórmula urgentibus imperii fatis, que ha mantenido dividida a la crítica. Nada promete a Roma su fin: ni la teoría de Tácito sobre la decadencia en general, ni su concepción del tiempo histórico, ni su opinión acerca del valor de los enemigos del Imperio ${ }^{81}$. La noticia de la Germania no se refiere evidentemente al presente: en el affo 98 , ya en tiempos del emperador Trajano, Tácito tenía menos razones que nunca para remover sombríos pensamientos, puesto que Roma había superado ya sus momentos de crisis más graves, representados por los desastres de sus ejércitos, la muerte de sus generales y la destrucción total de ciertas familias nobles ${ }^{2}$.

A pesar de todo se da cuenta de que, desde la invasión de los cimbrios, Roma habia sufrido importantes reveses frente a los germanos, entre los que se incluía el desastre fatal de Varo. Esto incidía en el hecho de que, desde hacía más de doscientos años, el Estado romano

\footnotetext{
78 Hist. IV 73. Cf. W. Steidle, «Tacitusprobleme», pp. 90-91.

79 Hist. IV 69: Nondum uictoria, iam discordia est.

80 Druso es aclamado por haber vencido a los germanos mediante discordias provocadas entre ellos: Ann. II 62.

81 M. P. Delpuech, \&Vrgentibus imperii fatis...», p. 1014.

82 Ann. III 6.
} 
trataba en vano de conquistar Germania; en consecuencia, el historiador podía esperar que, en tiempos de Trajano, diese comienzo una nueva era que viese acabar esta conquista del territorio germánico ${ }^{83}$. De ahí que el tema del final del Imperio romano exista en Tácito y que se halle expresado de manera ligeramente sospechosa en el discurso de Calgaco. Además, la idea de que el Imperio puede no ser eterno está expresada igualmente, a pesar de todas las razones de esperanza expuestas por Cerial, siendo esta misma idea la que encierra la frase final del capítulo que estamos analizando.

Sin embargo, todos los testimonios de Tácito que prevén con seriedad la destrucción del Imperio se encuentran en las Historias ${ }^{84}$. La frase final del texto de la Germania que estamos analizando constituye el resultado de una trágica experiencia vivida por Tácito en su juventud, la de la guerra civil del año 69 , siendo igualmente el resultado de otra experiencia distinta, la que dio al historiador romano, con la edad y la perspectiva del tiempo, el trato con el pasado y la reflexión sobre la historia. La idea de la decadencia del Imperio no se halla ausente, pues, de la obra tacitea, pero resulta extraño que la haya expresado en tiempos de Trajano: el ocaso de la elocuencia, la decadencia de las costumbres y de la civilización romana constituyen a la vez un bien y un mal.

Para tratar de encontrar una solución a las contradicciones inherentes a las cosas el método de Tácito se reviste de una casuística, a la que no escapa la suerte del Imperio: al reflexionar sobre la historia de Roma el historiador separa las condiciones necesarias para su final, es decir, la sincronización entre la discordia romana y la concordia de los bárbaros; de ahí que el análisis del mecanismo de las guerras civiles y del desarrollo del ansia de poder entre los hombres constituyesen para él razones de temor. Sin embargo, el estudio del peligro bárbaro le incitaba al optimismo, al demostrar el carácter crónico y la lógica interna de su desunión ${ }^{85}$.

En resumen, sólo las divisiones que hacían enfrentarse entre sí a los diferentes pueblos de Germania habían asegurado hasta entonces la pervivencia del poder romano, idea que, en cierto modo, reviste en Tácito un cierto carácter profético, puesto que de hecho cuando, algo más de un siglo después de plasmarla, los germanos se federaron, comenzará el verdadero ocaso de Roma. Por otro lado, si tenemos en cuenta sus origenes, formación y hechos más sobresalientes de su vida,

83 R. Syme, Tacitus, Oxford 1958, p. 126.

84 Hist. I 11, 30, 50 y 80; II 31 y 76; III 55 y 77 , y IV 13.

85 A. Michel, Tacite et le destin de l'Empire, Paris 1966, p. 65. 
no podemos extranarnos de que dicho historiador escribiese la Germania, ya que se trata de condenar en ella, por un lado, el lujo que había esclavizado a los romanos, así como la libertad excesiva que ocasionaba la perdición de sus enemigos y, por otro, de destacar las amenazas que se cernían sobre el Imperio y justificar al mismo tiempo la lealtad con respecto al poder.

Narciso Santos Yanguas 